Hydrology and Earth System Sciences, 9, 481-492, 2005

www.copernicus.org/EGU/hess/hess/9/481/

SRef-ID: 1607-7938/hess/2005-9-481

European Geosciences Union

(6)

\title{
A fast TDR-inversion technique for the reconstruction of spatial soil moisture content
}

\author{
S. Schlaeger ${ }^{1,2}$ \\ ${ }^{1}$ Soil Moisture Group (SMG), University of Karlsruhe, Germany \\ ${ }^{2}$ SCHLAEGER - mathematical solutions \& engineering, Karlsruhe, Germany
}

Received: 2 May 2005 - Published in Hydrology and Earth System Sciences Discussions: 13 June 2005

Revised: 6 September 2005 - Accepted: 23 September 2005 - Published: 13 October 2005

\begin{abstract}
Spatial moisture distribution in natural soil or other material is a valuably information for many applications. Standard measurement techniques give only mean or punctual results. Therefore a new inversion algorithm has been developed to derive moisture profiles along single TDR sensor-probes. The algorithm uses the full information content of TDR reflection data measured from one or both sides of an embedded probe. The system consisting of sensor probe and surrounded soil can be interpreted as a nonuniform transmission-line. The algorithm is based on the telegraph equations for nonuniform transmission-lines and an optimization approach to reconstruct the distribution of the capacitance and effective conductance along the transmission-line with high spatial resolution. The capacitance distribution can be converted into permittivity and water content by means of a capacitance model and dielectric mixing rules. Numerical investigations have been carried out to verify the accuracy of the inversion algorithm. Single- and double-sided time-domain reflection data were used to determine the capacitance and effective conductance profiles of lossless and lossy materials. The results show that singlesided reflection data are sufficient for lossless (or low-loss) cases. In case of lossy material two independent reflection measurements are required to reconstruct a reliable capacitance profile. The inclusion of an additional effective conductivity profile leads to an improved capacitance profile. The algorithm converges very fast and yields a capacitance profile within a sufficiently short time. The additional transformation to the water content requires no significant calculation time.
\end{abstract}

\section{Introduction}

The water content of soils and other porous materials is one of the most important parameters in hydrology, agriculture and civil engineering. Standard methods such as oven-drying are very time-consuming and destructive, neutron moderation or gamma attenuation measurements make use of critical radioactive sources. The determination of moisture content with time-domain reflectometry (TDR) technology is based on measurements of travel-time of an electromagnetic pulse on a transmission-line of known length. A review about TDR techniques for the measurement of permittivity and bulk electrical conductivity, but also for probe design and probe construction is given in Robinson et al. (2003). For homogeneous materials the travel-time is directly related to the permittivity, which is in common porous materials mainly a function of water content (Brichak et al., 1974; Topp et al., 1980, 1982a, b; Topp and Davis, 1985; Dasberg and Dalton, 1985).

One type of TDR transmission-line commonly used in many soil moisture relevant applications is an unshielded metallic fork, which is inserted into the material under test. The maximum length is limited, because the electromagnetic pulse is attenuated and disappears on longer lines. For longer transmission-line sensors insulated probes are more capable. The use of automation and multiplexing capability (i.e. Heimovaara and Bouten, 1990) increases the ability to monitor the dynamics and spatial distribution of water content.

So far TDR technology using single probes was limited to an integral or very coarsely resolved water content determination along the sensor line. But many applications ask for the spatial moisture distribution in soils or building constructions along a given profile. Different approaches have been developed to use TDR information for the determination of soil moisture profiles (multisection transmission lines: Hook et al., 1992; Feng et al., 1999; full wave

(info@stefan-schlaeger.de)

(C) 2005 Author(s). This work is licensed under a Creative Commons License. 


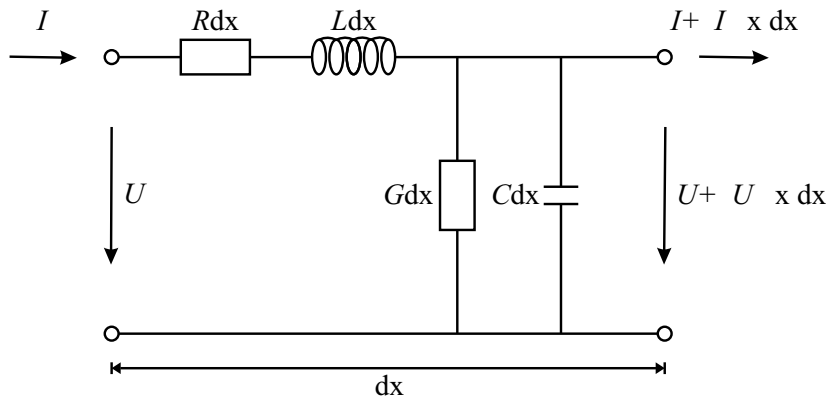

Fig. 1. Equivalent circuit of an infinitesimal section of a transverse electromagnetic (TEM) transmission-line.

inversion techniques: Pereira, 1997; Todoroff et al., 1998; Oswald, 2000; Heimovaara et al., 2004). A high spatial resolution can be achieved by exploiting the full information content in the reflected electromagnetic signals of the sensor line (Lundstedt and He, 1996; Norgren and He, 1996). A new reconstruction algorithm has been developed which uses the full information of the TDR signals measured on one or both sides of the line. It is based on the telegraph equation for nonuniform transmission-lines and an optimization approach to reconstruct simultaneously one or two line parameters with high spatial resolution (Schlaeger, 2001). In comparison with other full wave inversion techniques (e.g. generic algorithms, Oswald, 2000) it needs clearly less computation expenditure. The optimization algorithm uses the conjugate-gradient-technique and fast simplex or complex methods.

Using laboratory tests it is shown that TDR reflection data from both sides of a buried flat-ribbon-cable sensor are suitable for simultaneous reconstruction of capacitance and effective conductance profiles. During an investigation of the transport of volatile organic compounds in medium grained sand (grain size 0.2 to $1 \mathrm{~mm}$ ) the moisture profile under irrigation has been measured. In the steady state the volumetric water content varies along the vertically arranged transmission-line sensor of $71.7 \mathrm{~cm}$ between 0.5 and $44 \%$. The changes in water content could be reconstructed with a high spatial accuracy and an average uncertainty of $\pm 2.3 \%$ compared to oven-drying measurements (Schlaeger et al., 2005) $)^{1}$.

To determine moisture profiles, appropriate TDR-devices (short pulse rise time, high sampling rate) and sensitive testing probes (well known electric parameters) are required. The mathematical model has to be chosen to describe the physical process during the measurement in a very accurate and computable way. The inversion algorithm starts with an initial guess of the electric parameter distribution. Using this parameter distribution an associated TDR-signal can be calculated. The difference between the measurement and

\footnotetext{
${ }^{1}$ Schlaeger, S., Hübner, C., and Weber, K.: Moisture profile determination with TDR, in preparation, 2005.
}

this simulation leads to a rough deformation-instruction for the given parameter distribution. During one optimizationstep a new parameter distribution will be generated by deforming the old distribution according to the deformationinstruction. In the next step the comparison between measurement and simulation leads to an improved deformationinstruction. The optimization will be continued until a minimum difference is reached. The resulting electric parameter distribution can be easily transformed into water content profiles. The whole process is called Spatial-TDR.

The Soil Moisture Group (SMG) at the University of Karlsruhe has tested this Spatial-TDR technology in many applications. A monitoring system to measure the spatial soil water distribution on a full-scale levee model has been successfully implemented with transmission-lines up to $3 \mathrm{~m}$ and leads to significant specifications for drainage models (Scheuermann et al., 2001). The technology is also being used for flood warning systems, and snow moisture measurements (Becker et al., 2002; Stacheder et al., 2005), and for the determination of the water content of technical barriers in waste disposal sites (Becker et al., 2003).

\section{Basic equations}

The propagation of electromagnetic waves on insulated and non insulated transmission-lines can be described by the telegraph equations. These equations were developed by Heaviside in 1886. In this model the transmission-line is characterized by four electrical parameters: the inductance $L$, capacitance $C$, series resistance $R$, and shunt conductance $G$. The equivalent electric circuit of an infinitesimal transmissionline section is given in Fig. 1. It is seen that the inductance and resistance are series elements that cause a voltage drop along the line, whereas the capacitance and conductance are shunt elements that provide a current path between the conductors.

From a circuit theory approach it is a simple matter to derive the telegraph equations that describe the variation of the voltage $U(x, t)$ and the current $I(x, t)$ in the time along the transmission-line due to the influence of the electric parameters of the line and the surrounding media. By applying Kirchhoff's voltage and current laws to the equivalent circuit in Fig. 1, one obtains

$$
\begin{gathered}
\frac{\partial}{\partial x} U(x, t)=-R(x) I(x, t)-L(x) \frac{\partial}{\partial t} I(x, t), \\
\frac{\partial}{\partial x} I(x, t)=-G(x) U(x, t)-C(x) \frac{\partial}{\partial t} U(x, t) .
\end{gathered}
$$

Usually $R$ and $L$ are constant for the probe whereas $C$ and $G$ depend on the surrounding material. In most cases $R$ can be neglected. The conductance $G$ of transmission-line sensors embedded in soil depends on soil type, water content, and frequency. Usually clayey and loamy soils have much 


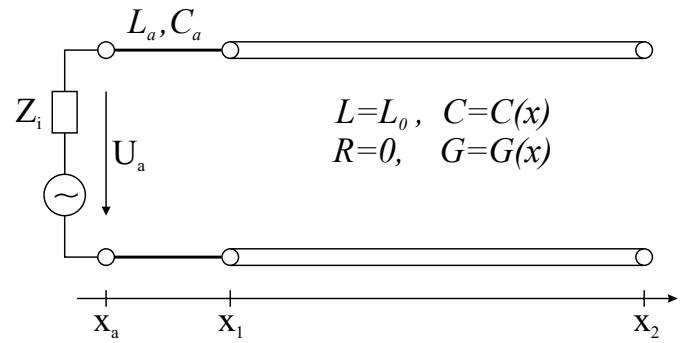

Fig. 2. Schematic representation of a sensitive transmission-line, situated between $x_{1}$ and $x_{2}$, which is connected to a TDR-device on one side using a lossless uniform coaxial-cable with impedance $Z=\left(L_{a} / C_{a}\right)^{0.5}$.

higher conductivity than sands. The capacitance $C$ is intimately connected with the permittivity and therefore the water content of the surrounding medium. The determination of the spatial distribution of $C(x)$ is the key component of the presented reconstruction of the soil water content.

The solution of Eqs. (1) and (2) describes the propagation in time and space of a supplied pulse to the whole measurement system. When the solution is restricted to one spatial point it represents a simulated measurement in this point. To calculate the solution initial and boundary conditions for the partial differential equations (PDE) are needed. It is also important to consider the connection between TDR-device and testing probe. Usually they are connected with a lossless and uniform coaxial-cable ( $R=0, G=0, C=$ const, and $L=$ const). Assume that there is no energy on the line at the beginning of the measurement. So the initial conditions can be set to

$U(x, t)_{t \leq 0}=0, \quad I(x, t)_{t \leq 0}=0$, for all $x$.

Than the Eqs. (1) and (2) can be transformed to one single PDE of second order

$$
\left[L C \frac{\partial^{2}}{\partial t^{2}}+L G \frac{\partial}{\partial t}+\frac{\partial L / \partial x}{L} \frac{\partial}{\partial x}-\frac{\partial^{2}}{\partial x^{2}}\right] U(x, t)=0 .
$$

The derivative of $L$ has to be considered in Eq. (4) because the inductance of the coaxial-cable and the testing probe are constant but may be different in general. The initial conditions (Eq. 3) can be transformed to

$$
\left.U(x, t)\right|_{t \leq 0}=0,\left.\frac{\partial}{\partial t} U(x, t)\right|_{t \leq 0}=0 \text {, for all } x .
$$

To define the boundary conditions for the PDE (Eq. 4) the whole measurement configuration has to be considered. The sensitive transmission-line has to be inserted into the soil and must be connected to a TDR-device in order to excite an electric pulse.

Figure 2 describes the experimental setup to receive the reflection data from one side of the sensitive transmission-line. Therefore reflection measurements must be realized with an external current $F_{e x}=\delta(x-x)_{a} \cdot f(t)$ at $x=x_{a}$. The backtraveling wave is absorbed by the matched impedance $Z_{i}$

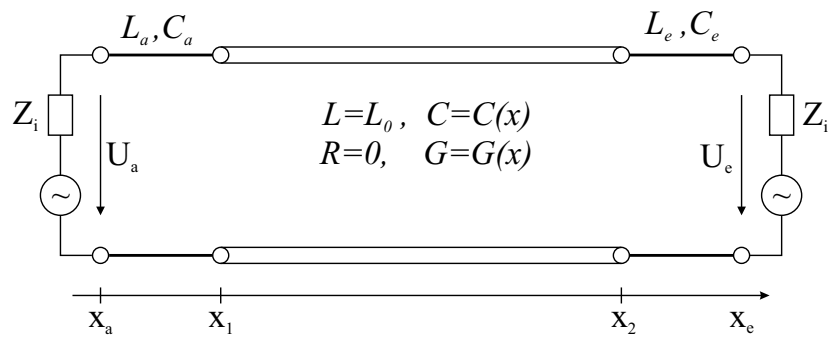

Fig. 3. The nonuniform transmission-line, situated between $x_{1}$ and $x_{2}$, is connected to two lossless uniform coaxial-cables with matched impedances $Z_{i}$ at their endpoints.

inside the TDR-device if it is equal to the impedance $Z$ of the coaxial-cable. This absorbing boundary condition for the lossless wave equation can be numerically implemented in the coaxial-cable using (Engquist and Majda, 1977):

$$
\left[\frac{\partial}{\partial x}-\sqrt{L_{a} C_{a}} \frac{\partial}{\partial t}\right] U\left(x_{a}, t\right)=L_{a} \frac{\partial}{\partial t} F_{e x}\left(x_{a}, t\right), \quad t \geq 0 .
$$

The boundary conditions at the end of the sensitive line at $x=x_{2}$ depends on its physical implementation. In case of an open-circuit the boundary condition will be

$$
\frac{\partial}{\partial t} U\left(x_{2}, t\right)=0, \quad t \geq 0 .
$$

If there is a short-circuit at $x=x_{2}$ the boundary condition will be $U\left(x_{2}, t\right)=0$, for $t \geq 0$.

In order to reconstruct two parameters, two independent measurements are needed. Consequently, the problem is divided into two parts, the first dealing with an incident wave from the left and the second with an incident wave from the right side of the system under test.

Figure 3 describes the experimental setup to receive the reflection data from both sides of the unknown material. Therefore two separate measurements must be realized with the external current $F_{e x}^{1}=\delta\left(x-x_{a}\right) \cdot f(t)$ and $F_{e x}^{2}=\delta\left(x-x_{e}\right) \cdot f(t)$, respectively. $U_{1}(x, t)$ and $U_{2}(x, t)$ are the solutions of both separate forward problems. The setup of Fig. 3 can be transformed to the setup in Fig. 2 for each single-sided measurement using sensor switches (Becker and Hübner, 2003). In this case the solution of $U_{1}(x, t)$ can be calculated according to $U(x, t)$ by using Eqs. (4)-(7). If there is a coaxial-cable permanently attached at $x=x_{e}$ an absorbing boundary condition has to be used instead of Eq. (7):

$\left[\frac{\partial}{\partial x}+\sqrt{L_{e} C_{e}} \frac{\partial}{\partial t}\right] U_{1}\left(x_{e}, t\right)=0, \quad t \geq 0$

For the other initial-boundary-value-problem (IBVP) for $U_{2}$ with external current $F_{e x}^{2}$ the boundary conditions are exchanged.

Before the reconstruction procedure can be started all necessary parameters $\left(L_{a}, C_{a}, L_{e}, C_{e}, L_{0}\right)$ have to be measured or calculated (Becker, 2004; Becker and Schlaeger, 2005). 
The inverse method presented in the next section is based on an iterative search for the electrical parameters of the nonuniform transmission-line with the full wave solution of the direct problem. The solution of the line needs to be calculated repetitively. It is therefore important to use a technique for the determination of this solution that is computationally efficient to guarantee low calculating time.

\section{Optimization approach}

The aim of the investigation is the determination of the unknown distribution of $C(x)$ with measurements of input and output data. The input data $f(t)$, which describes the incident pulse, can be easily determined from the reflection measurements $U_{a}\left(x_{a}, t\right)$ of the coaxial-cable between $x_{a}$ and $x_{1}$ with an open-circuit at $x=x_{1}$. The output data $\lambda(t)$ is the reflected signal based on the associated input signal at one side of the sensor line.

The cost function $J(C)$ defines the squared difference (in $L_{2}$-norm) between the solution of the direct problem (Eqs. 4 7) restricted to $x=x_{1}$ corresponding to one given parameter distribution $C$ and the measured reflections $\lambda(t)$ at $x=x_{1}$,

$J(C)=\left\|U\left(x_{1}, t ; C\right)-\lambda(t)\right\|_{2}^{2}=\int_{0}^{2 T}\left[U\left(x_{1}, t ; C\right)-\lambda(t)\right]^{2} d t(9)$

with $T=\tau\left(x_{1}, x_{2}\right)$, where $\tau\left(x_{1}, x_{2}\right)$ is the travel-time between $x_{1}$ and $x_{2}$. The cost function refers to the error in the solution for single-sided incidence measurements. The concept of the method is to find the parameter distribution that minimize the cost functional $J$. If the problem has a solution the theoretical minimum of $J$ is zero. One important reason for choosing the $L_{2}$-norm is the possibility to derive exact expressions for the gradient of $J$.

\subsection{Exact expression of the gradient of the cost function}

In the following section the gradient of the cost function will be determined for single-sided and double-sided reflection data, respectively. For one single measurement it is only possible to calculate one parameter distribution $C(x)$ or $G(x)$ while the other one is known (He et al., 1993). Assuming that the capacitance and conductance are connected by a transfer function then it is possible to calculate both parameter distributions from one single reflection measurement using an empirical $G(C)$-relationship (Hakansson, 1997). This relationship depends not only on the water content but also on the electrolyte imbalance of the pore water. The determination of this relation may be very time intensive (Becker, 2004; Becker and Schlaeger, 2005). If both parameter distributions are to be calculated simultaneously without this $G(C)$-relationship, two independent measurements have to be carried out, e.g. double-sided reflection measurements (He et al., 1994). In the first case the gradient for
$J(\alpha)=J(C)$ or $J(\alpha)=J(G)$, in the second case the gradient for $J(\alpha)=J(C, G)$ is calculated. In both cases the gradient $\nabla J(\alpha)$ can be calculated using the standard finite difference formulation for $\delta \alpha \rightarrow 0$ :

$$
\begin{aligned}
& J(\alpha+\delta \alpha)-J(\alpha)=\langle\delta \alpha, \nabla J(\alpha)\rangle= \\
& \int_{-\infty}^{\infty} \delta \alpha(x) \cdot \nabla J(\alpha)(x) d x .
\end{aligned}
$$

\subsubsection{Single-sided reflection data}

In the case of only one single-sided reflection data-set it is reasonable to reconstruct the capacitance profile $C(x)$ to determine the water content. Therefore the information about the gradient $\nabla J(C)$ has to be generated. According to Eq. (9), Eq. (10) can be transferred to

$$
\begin{aligned}
J & (C+\delta C)-J(C) \\
= & \int_{0}^{2 T}\left[U\left(x_{1}, t ; C+\delta C\right)-\lambda(t)\right]^{2}-\left[U\left(x_{1}, t ; C\right)-\lambda(t)\right]^{2} d t \\
= & \int_{0}^{2 T} U^{2}\left(x_{1}, t ; C+\delta C\right)-U^{2}\left(x_{1}, t ; C\right) \\
& -2 \lambda(t)\left[U\left(x_{1}, t ; C+\delta C\right)-U\left(x_{1}, t ; C\right)\right] d t \\
= & \int_{0}^{2 T}[\underbrace{\left[U\left(x_{1}, t ; C+\delta C\right)+U\left(x_{1}, t ; C\right)\right]}_{=: \delta U\left(x_{1}, t ; C\right)} . \\
& \underbrace{\left[U\left(x_{1}, t ; C+\delta C\right)-U\left(x_{1}, t ; C\right)\right]}_{-2 \lambda\left(x_{1}, t ; C\right)} \\
\approx & \int_{0}^{2 T} 2 \delta U\left(x_{1}\left(x_{1}, t ; C\right) \cdot\left[U\left(x_{1}, t ; C\right)-\lambda(t)\right] d t\right. \\
= & \int_{0}^{2 T} \int_{-\infty}^{\infty} \delta U(x, t ; C) \cdot 2 \delta\left(x-x_{1}\right)[U(x, t ; C)-\lambda(t)] d x d t .
\end{aligned}
$$

In Eq. (11) $\delta(x)$ represents the Dirac delta-function with $\int \delta\left(x-x_{0}\right) f(x)=f\left(x_{0}\right)$ for all $f$. The difference between two solutions according to a difference between $C$ and $C+\delta C$ is defined by $\delta U(C)=U(C+\delta C)-U(C)$. For small discrepancies in $C$ the difference $\delta U(C)$ is assumed to be also small and $U(C+\delta C)+U(C) \approx 2 U(C)$. To make further transformations of Eq. (11) the advantages of adjoint operators will be used. Therefore a linear operator $\boldsymbol{L}$ is defined analog to Eq. (4):

$$
\boldsymbol{L} U \equiv\left[L C \frac{\partial^{2}}{\partial t^{2}}+L G \frac{\partial}{\partial t}+\frac{\partial L / \partial x}{L} \frac{\partial}{\partial x}-\frac{\partial^{2}}{\partial x^{2}}\right] U
$$

The definition of the adjoint operator $\boldsymbol{L}^{*}$ is that he will fulfil the following equation for every $U(x, t)$ and $V(x, t)$

$$
(\boldsymbol{L} U \mid V)=\left(U \mid \boldsymbol{L}^{*} V\right)
$$


using the inner product

$$
(U \mid V)=\int_{0}^{2 T} \int_{-\infty}^{\infty} U(x, t) V(x, t) d x d t .
$$

Now the PDE for the adjoint operator $\boldsymbol{L}^{*}$ has to be determined. The left side of Eq. (13) can be transformed using Eqs. (12) and (14). Each of the double-integrals will be transformed to isolate $U(x, t)$ using integration by parts in every addend. The auxiliary terms resulting from this isolation must be eliminated now, as the initial and boundary conditions for $V$ are appropriate selected. The notation $U_{t}$ and $U_{t}$ in the following equation is an abbreviation for the partial differential derivative $\partial U / \partial t$ and $\partial^{2} U / \partial t^{2}$, respectively.

$$
\begin{aligned}
& (\boldsymbol{L} U \mid V) \\
& =\int_{0}^{2 T} \int_{-\infty}^{\infty} L C U_{t t} V d x d t+\int_{0}^{2 T} \int_{-\infty}^{\infty} L G U_{t} V d x d t \\
& +\int_{0}^{2 T} \int_{-\infty}^{\infty} \frac{L_{x}}{L} U_{x} V d x d t-\int_{0}^{2 T} \int_{-\infty}^{\infty} U_{x x} V d x d t \\
& =\int_{-\infty}^{\infty} L C\left(\left[U_{t} V\right]_{t=0}^{t=2 T}-\left[U V_{t}\right]_{t=0}^{t=2 T}+\int_{0}^{2 T} U V_{t t} d t\right) d x \\
& +\int_{-\infty}^{\infty} L G\left([U V]_{t=0}^{t=2 T}-\int_{0}^{2 T} U V_{t} d t\right) d x \\
& +\int_{0}^{2 T} \\
& \left(\left[U \frac{L_{x}}{L} V\right]_{x=-\infty}^{x=\infty}-\int_{-\infty}^{\infty} U\left(\frac{L_{x}}{L}\right)_{x} V d x-\int_{-\infty}^{\infty} U \frac{L_{x}}{L} V_{x} d x\right) d t \\
& -\int_{0}^{2 T}\left(\left[U_{x} V\right]_{x=-\infty}^{x=\infty}-\left[U V_{x}\right]_{x=-\infty}^{x=\infty}+\int_{-\infty}^{\infty} U V_{x x} d x\right) d t \\
& \begin{aligned}
\stackrel{\# 1}{=} \int_{0}^{2 T} \int_{-\infty}^{\infty} L C V_{t t} U d x d t-\int_{0}^{2 T} \int_{-\infty}^{\infty} L G V_{t} U d x d t \\
\quad-\int_{0}^{2 T} \int_{-\infty}^{\infty} \frac{L_{x}}{L} V_{x} U d x d t-\int_{0}^{2 T} \int_{-\infty}^{\infty} V_{x x} U d x d t
\end{aligned} \\
& \stackrel{\# 2}{=}\left(U \mid \boldsymbol{L}^{*} V\right)
\end{aligned}
$$

To make sure that transformation \#1 in Eq. (15) is correct the initial and boundary conditions for $V(x, t)$ have to be set as follows

$$
\begin{aligned}
& V(x, 2 T)=0, \frac{\partial}{\partial t} V(x, 2 T)=0,-\infty<x<\infty, \\
& V(-\infty, t)=0, \frac{\partial}{\partial t} V(\infty, t)=0,0 \leq t \leq 2 T .
\end{aligned}
$$

The initial condition results to a backward propagation in time from $2 T$ to zero. When the operator $L^{*}$ is defined by

$$
\boldsymbol{L}^{*} V \equiv\left[L C \frac{\partial^{2}}{\partial t^{2}}-L G \frac{\partial}{\partial t}-\frac{\partial L / \partial x}{L} \frac{\partial}{\partial x}-\frac{\partial^{2}}{\partial x^{2}}\right] V
$$

then equivalence \#2 in Eq. (15) will also be fulfilled. Now the adjoint operator $\boldsymbol{L}^{*}$ that accomplishes Eq. (13) is found with its corresponding PDE (Eq. 18) and initial and boundary conditions (Eqs. 16-17). The solution $V$ of this PDE can only be different from zero if a non vanishing right side is assigned to $\boldsymbol{L}^{*} V$. Looking back to Eq. (11) one can equate

$\boldsymbol{L}^{*} V=2 \delta\left(x-x_{1}\right)[U(x, t ; \alpha)-\lambda(t)]$

to continue the transformations of Eq. (11) and use the property of $\boldsymbol{L}^{*}$ being the adjoint operator to $\boldsymbol{L}$ according to the inner product (Eq. 14).

$$
\begin{aligned}
& J(C+\delta C)-J(C) \\
& =\int_{0}^{2 T} \int_{-\infty}^{\infty} \delta U \cdot\left(\boldsymbol{L}^{*} V\right) d x d t \\
& =\int_{0}^{2 T} \int_{-\infty}^{\infty}(\boldsymbol{L} \delta U) \cdot V d x d t \\
& 2 T \infty \\
& =\int_{0} \int_{-\infty} V \\
& {\left[L \delta C U_{t t}+L C \delta U_{t t}+L G \delta U_{t}+\frac{L_{x}}{L} \delta U_{x}-\delta U_{x x}\right] d x d t} \\
& =\int_{-\infty}^{\infty} \delta C \int_{0}^{2 T} V L U_{t t} d t d x \\
& +\underbrace{\int_{0}^{2 T} \int_{-\infty}^{\infty} V\left[L C \delta U_{t t}+L G \delta U_{t}+\frac{L_{x}}{L} \delta U_{x}-\delta U_{x x}\right] d x d t}_{\rightarrow 0 \text { for }\|\delta C\| \rightarrow 0}
\end{aligned}
$$

From Eqs. (20) and (10) it follows that

$\nabla J(C)=\int_{0}^{2 T} L V \frac{\partial^{2}}{\partial t^{2}} U d t=-\int_{0}^{2 T} L \frac{\partial}{\partial t} V \frac{\partial}{\partial t} U d t$.

This gradient can be calculated by solving two IBVP: One forward problem for the direct wave (Eqs. 4-7) and one backward problem for the adjoint wave (Eqs. 16-19).

\subsubsection{Double-sided reflection data}

For the simultaneous reconstruction of $C(x)$ and $G(x)$ it is necessary to take two independent measurements. As shown in the previous section one choice will be the two reflection measurements from both sides of the sensor:

$\lambda_{1}(t)=U_{a}\left(x_{1}, t\right), \lambda_{2}(t)=U_{e}\left(x_{2}, t\right)$

It is also necessary to choose another cost function in order to minimize the error between the simulation and the measurements simultaneously. Choosing $J(\alpha)=J(C, G)$ as

$J(\alpha)=\sum_{i=1}^{2} \int_{0}^{2 T}\left[U_{i}\left(x_{i}, t ; \alpha\right)-\lambda_{i}(t)\right]^{2} d t$. 


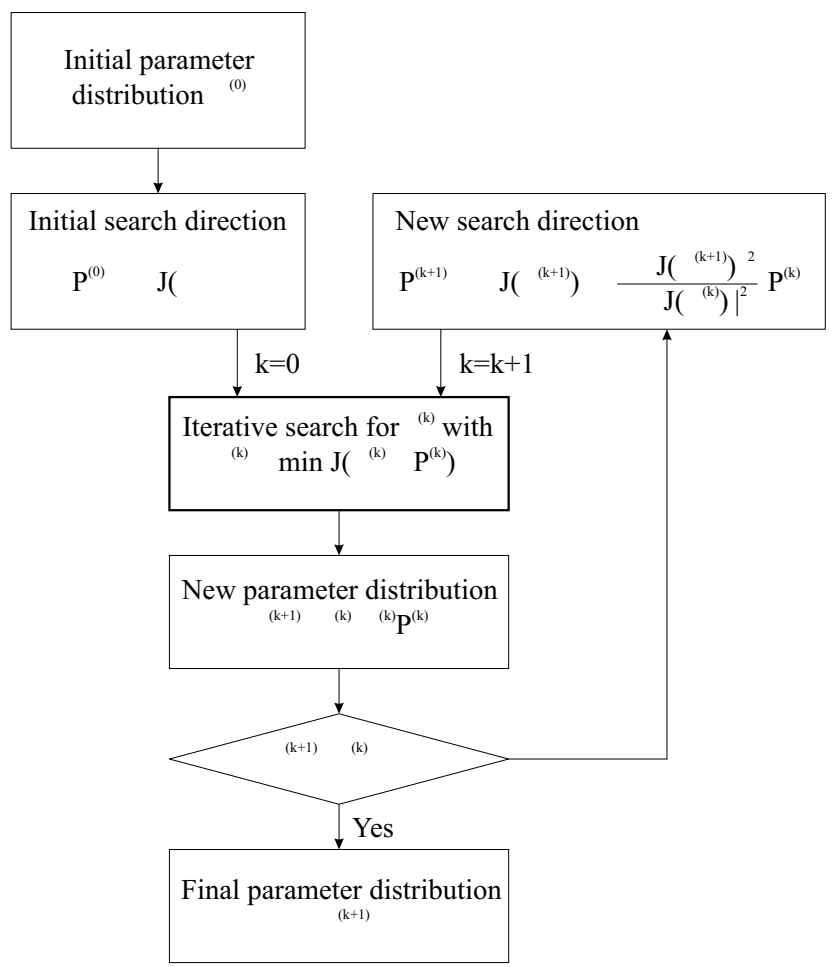

Fig. 4. Flow chart of the Fletcher-Reeves conjugate gradient method.

The determination of the two gradients is very similar to the transformations above. It leads to the same adjoint PDE for $\boldsymbol{L}^{*}$ as in Eq. (18). But two different backward problems have to be calculated according to the two different forward solutions $U_{1}$ and $U_{2}$. The right sides of the adjoint problems are given by

$\boldsymbol{L}^{*} V_{i}=2 \delta\left(x-x_{i}\right)\left[U_{i}(x, t ; \alpha)-\lambda_{i}(t)\right], i=1,2$

and the initial and boundary conditions have to be chosen to fulfil

$V_{i}(x, 2 T)=0, \frac{\partial}{\partial t} V_{i}(x, 2 T)=0,-\infty<x<\infty, i=1,2$,

$V_{i}(-\infty, t)=0, \frac{\partial}{\partial t} V_{i}(\infty, t)=0,0 \leq t \leq 2 T, i=1,2$.

For each given $\alpha=(C(x), G(x))$ one can solve the forward problems for $U_{1}(x, t)$ and $U_{2}(x, t)$ and the backward problems for $V_{1}(x, t)$ and $V_{2}(x, t)$ and calculate the gradients of $J(\alpha)$ with respect to $C$ and $G$, respectively:

$\nabla^{C} J(\alpha)=-\sum_{i=1}^{2} \int_{0}^{2 T} L \frac{\partial}{\partial t} V_{i} \frac{\partial}{\partial t} U_{i} d t$

$\nabla^{G} J(\alpha)=\sum_{i=1}^{2} \int_{0}^{2 T} L V_{i} \frac{\partial}{\partial t} U_{i} d t$

\subsection{Reconstruction of the parameter distribution}

To determine the distribution of $\alpha=C(x)$ a conjugate gradient $(\mathrm{cg})$ method is appropriate if the gradient of the function to be minimized can be calculated explicitly and very easily. Starting with a parameter distribution $\alpha^{(0)}$ the first search direction is given by the direction of steepest decent $P^{(0)}=-\nabla J\left(\alpha^{(0)}\right)$. The next parameter distribution can be calculated by

$\alpha^{(k+1)}=\alpha^{(k)}+\gamma^{(k)} P^{(k)}$

where $\gamma^{(k)}$ is the optimal step-size to minimize the cost function based on the former parameter distribution $\alpha^{(k)}$ and the search-direction $P^{(k)}$ :

$\gamma^{(k)}=\min _{\gamma} J\left(\alpha^{(k)}+\gamma \cdot P^{(k)}\right)$.

The main effort of computation in this minimization is due to the large number of cost function analysis. Therefore an effective numerical algorithm concerning minimum function calls is the core of a fast reconstruction algorithm. In comparison to conventional $\mathrm{cg}$-methods the following search direction $P^{(k+1)}$ is not only the direction of steepest decent at $\alpha^{(k+1)}$ but also a combination of former search-directions.

$P^{(k+1)}=-\nabla J\left(\alpha^{(k+1)}\right)+\frac{\left\|\nabla J\left(\alpha^{(k+1)}\right)\right\|_{2}^{2}}{\left\|\nabla J\left(\alpha^{(k)}\right)\right\|_{2}^{2}} \cdot P^{(k)}$

This leads to a faster convergence and less calculation effort. The cg-method was chosen according to Fletcher and Reeves (1964) - similar results were carried out by using the cgmethod according to Polak and Ribière (1969).

During simultaneous reconstruction of $C(x)$ and $G(x)$ the minimum search in Eq. (30) will be extended to a twodimensional search:

$$
\left(\gamma^{(k)}, \eta^{(k)}\right)=\min _{\gamma, \eta} J\left(C^{(k)}+\gamma \cdot P_{C}^{(k)}, G^{(k)}+\eta \cdot P_{G}^{(k)}\right)
$$

Especially in this two-dimensional search the choice of an algorithm with as few function calls as possible is of crucial importance. The simplex method developed by Nelder and Mead (1965) and the complex method by Box (1965) lead to fast optimization algorithms even in the simultaneous reconstruction of two parameter functions. At one- and two-parameter optimization the conjugate gradient algorithm terminates if there is no significant change in the value of two consecutive cost functions. Figure 4 shows the flow chart of the preferred cg-method.

When using two measurements which show only small differences in the reflected signal than the corresponding calculated parameter distribution shows only small variations as well. 


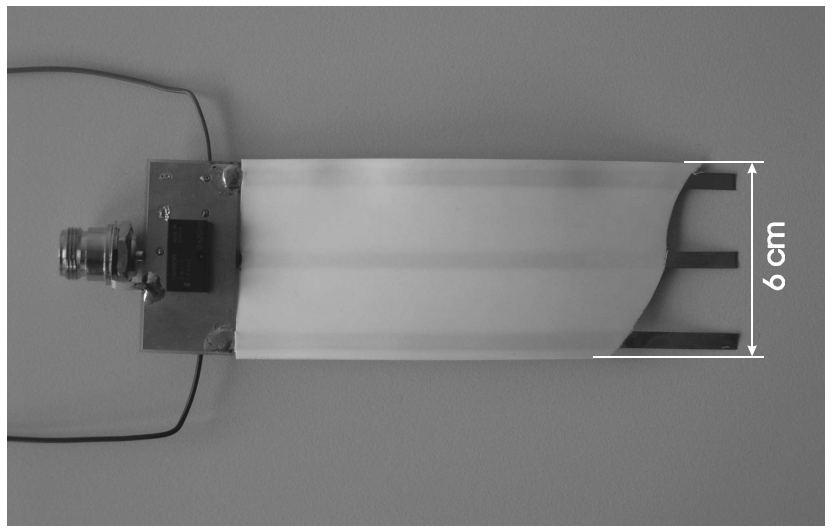

Fig. 5. Insulated flat-ribbon-cable (short section with bare conductors to visualize the geometry and the electrical connection of the cable) with a sensor switch between coaxial cable and flat-ribboncable.

Table 1. Cable parameters for the sensor cable in Fig. 5.

\begin{tabular}{lcccc}
\hline Circuit element & $\begin{array}{c}C_{1} \\
(\mathrm{pF} / \mathrm{m})\end{array}$ & $\begin{array}{c}C_{2} \\
(\mathrm{pF} / \mathrm{m})\end{array}$ & $\begin{array}{c}C_{3} \\
(\mathrm{pF} / \mathrm{m})\end{array}$ & $\begin{array}{c}L_{0} \\
(\mathrm{nH} / \mathrm{m})\end{array}$ \\
\hline Measured value & 3.4 & 323 & 14.8 & 756 \\
Calculate value & 4.0 & 308 & 13.7 & 785 \\
\hline
\end{tabular}

\section{Determination of the water content}

The capacitance profile $C(x)$ describes the electrical properties of the whole medium around the conductors of the sensitive transmission-line. But strictly speaking, the capacitance at any point of the profile is not independent on the frequency. For simplification, we assume that there may be a constant value for $C$ for the used TDR frequency range depending on feeding cable properties and length. If the sensor is not insulated $C(x)$ can be easily transformed into the relative permittivity of the medium $\varepsilon_{m}(x)=L_{0} c_{0}^{2} C(x)$, where $L_{0}$ specifies the inductance of the sensor and $c_{0}$ the speed of light in vacuum. If the sensor is insulated with some dielectric material the total capacitance $C(x)$ represents the combination of insulation and soil. Therefore a sensor specific transformation from $C$ to the relative permittivity $\varepsilon$ of the soil is necessary.

The flat-ribbon-cable used for many applications of the SMG is shown in Fig. 5. It has been developed and patented by the Institute of Meteorology and Climate Research at the Forschungszentrum Karlsruhe (Brandelik et al., 1998). The cable consists of three flat copper wires covered with polyethylene. The electrical field is concentrated around the conductors and defines the sensitive area of 3 to $5 \mathrm{~cm}$ around the cable depending on the permittivity. The electric properties of the flat-ribbon-cable used in this work can be mea-

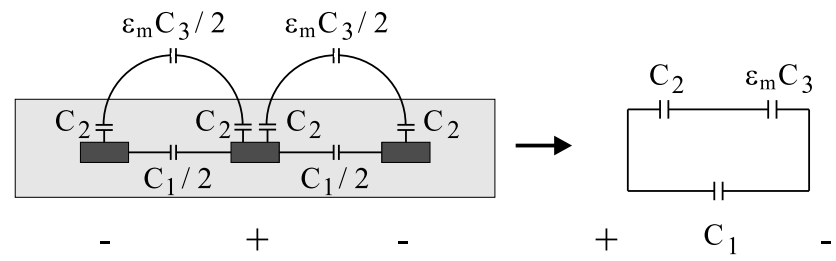

Fig. 6. Capacitance model of the insulated flat-ribbon-cable from Fig. 5 .

sured and calculated (cf. Fig. 6 and Table 1).

According to the equivalent circuit of Fig. 6 the total capacitance $C$ can be expressed by three capacitances $C_{1}, C_{2}$, and $\varepsilon_{m} C_{3}$ and can be transformed into a direct relation between the relative permittivity $\varepsilon_{m}$ of the surrounding soil and the total capacitance:

$C=C_{1}+\frac{C_{2} \varepsilon_{m} C_{3}}{C_{2}+\varepsilon_{m} C_{3}}$

The three unknown capacitances $C_{1}, C_{2}$, and $C_{3}$ were derived from calibration measurements of three different materials with well known dielectric properties, e.g. air, oil, and water. The inductance was determined by measuring the wave impedance with a variable resistor at the end of the cable adjusted for minimum reflection. The values for the cable of Fig. 5 are given below (Hübner, 1999).

The permittivity of the soil can now be transformed to the volumetric water content by standard transformations for arbitrary soils (e.g. Topp et al., 1980) or soil specific calibration functions determined from laboratory test series. The accuracy of the water content distribution depends highly on the accuracy of this transformation. The deviation due to insufficient knowledge of the material can easily exceed the errors of the reconstruction of the capacitance profile $C(x)$.

The total conductance $G(x)$ describes the conductivity of the material between the copper wires, i.e. the system of polyethylene insulation and the surrounding material. The determination of the water content distribution of the surrounding material does not require the knowledge of the conductivity distribution of the material, but it cannot be neglected during the reconstruction of $C(x)$.

\section{Numerical results}

In order to investigate the accuracy of the reconstruction with respect to a known profile, artificial time-domain reflection data have been generated to provide input and output data for single- and double-sided reconstructions of the capacitance profile. Therefore a $2 \mathrm{~m}$ flat-ribbon-cable sensor was surrounded by several sections of different material with given electrical properties. Two different capacitance and conductance profile combinations were used to represent one lossless and one lossy soil profile (see sections of constant electric properties given in Table 2). In contrast to the abrupt 
Table 2. Electrical properties of the test materials to represent lossless and lossy material.

\begin{tabular}{llcccc}
\hline \multicolumn{5}{c}{ Position $(\mathrm{m})$} \\
\cline { 3 - 6 } & & $0.0-0.3$ & $0.3-1.3$ & $1.3-1.7$ & $1.7-2.0$ \\
\hline \multirow{2}{*}{ Lossless } & $\mathrm{C}(\mathrm{pF} / \mathrm{m})$ & 20 & 40 & 80 & 20 \\
& $\mathrm{G}(\mathrm{mS} / \mathrm{m})$ & 0 & 0 & 0 & 0 \\
\hline \multirow{2}{*}{ Lossy } & $\mathrm{C}(\mathrm{pF} / \mathrm{m})$ & 20 & 40 & 80 & 20 \\
& $\mathrm{G}(\mathrm{mS} / \mathrm{m})$ & 0 & 4 & 8 & 0 \\
\hline
\end{tabular}

changes of this synthetic example natural soil profiles show smooth transients in the water content (related to the chosen spatial discretization step).

These parameter distributions lead to left- and right-sided reflection data for the lossless and lossy material (Hübner et al., 2005), see Fig. 7.

The initial capacitance $C_{0}=\tau^{2}\left(x_{1}, x_{2}\right) /\left(L_{0}\left(x_{2}-x_{1}\right)^{2}\right)$ can be easily determined by simple travel-time measurements along the cable sensor (Heimovaara and Bouten, 1990). To ensure the invariance of this sensor travel-time during the conjugate gradient algorithm one has find a constant shift $C_{\gamma}$ for every given $\gamma$ during the optimization to fulfil

$\sqrt{C_{0}}\left(x_{2}-x_{1}\right)=\int_{x_{1}}^{x_{2}} \sqrt{C(x)+\gamma P_{C}+C_{\gamma}} d x$

This shift correction guarantees that all determined capacitance profiles lead to the same total travel-time along the cable sensor. It means that the mean moisture content remains invariant during the optimization. Therefore the single roundtrip travel-time $\tau\left(x_{1}, x_{2}\right)$ has to be determined as accurate as possible. Equation (29) has to be modified to get the advanced consecutive capacitance profile in the cgalgorithm:

$C^{(k+1)}=C^{(k)}+\gamma^{(k)} P^{(k)}+C_{\gamma^{(k)}}$

with

$\gamma^{(k)}=\min _{\gamma} J\left(C^{(k)}+\gamma \cdot P^{(k)}+C_{\gamma}\right)$.

\subsection{One-parameter reconstruction}

Compared to lossy materials the reconstruction of the true capacitance profile in lossless soil $(G(x)=0)$ is rather simple. Only one unknown parameter distribution $C(x)$ has to be determined. Therefore one single reflection measurement is sufficient to derive the final capacitance profile.

To calculate the wave propagation the cable sensor is separated into 400 equally spaced sections of $5 \mathrm{~mm}$. For this discretization the single-sided reconstruction algorithm needs about 15 min on a standard PC to calculate 20 iteration steps. The similar reconstruction using right-sided reflection data leads to comparable results.
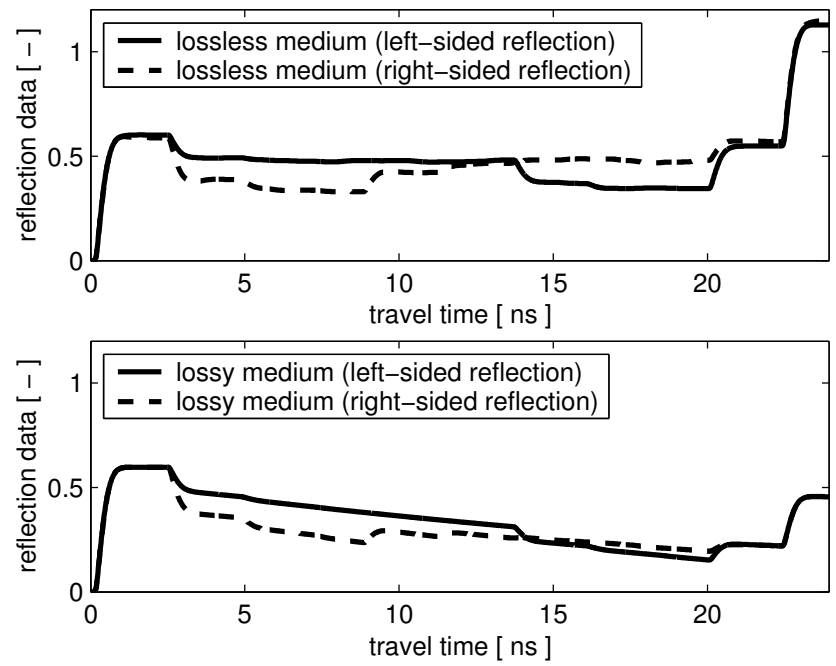

Fig. 7. Left- and right-sided reflection data for the lossless (above) and lossy (below) soil profile, both with main reflections at $22.3 \mathrm{~ns}$ as a result of an open-circuit at the end of the cable sensor.

Figure 8 shows several intermediate results during the conjugate gradient optimization. The search direction leads to a good approximation of the capacitance profile very fast. After the first iteration the main features are mapped very well. The following iterations lead to smaller corrections of rather fine structures.

In the case of lossy material with an unknown effective conductance profile $G(x)$ a first approach is to reconstruct $C(x)$ using single-sided reflection data and assume $G(x)$ to be equal to zero over the whole area. Figure 9 show the results of this reconstruction. The capacitance is overestimated due to the effect of the non vanishing conductivity. Furthermore the optimization stops after three iteration steps, because the calculated search direction could not improve the value of the cost function (Eq. 9).

This distortion is reduced if the mean value of the conductance will be used which can also be determined from the time domain reflection. A mean value of $G(x)=4 \mathrm{mS} / \mathrm{m}$ was chosen to estimate the effect of the conductivity. Figure 10 shows the reconstruction of the capacitance using this assumption.

The results of the capacitance reconstruction presented in Figs. 9 and 10, do not satisfy the expectations on a reliable solution of the soil moisture determination. To get better results it is necessary to use additional information to reconstruct the capacitance $C(x)$ and the conductance $G(x)$ simultaneously.

\subsection{Two-parameter reconstruction}

To reconstruct $C(x)$ and $G(x)$ simultaneously, two independent measurements are needed. In the case of the lossy soil described in Table 2, reflection measurements from both 

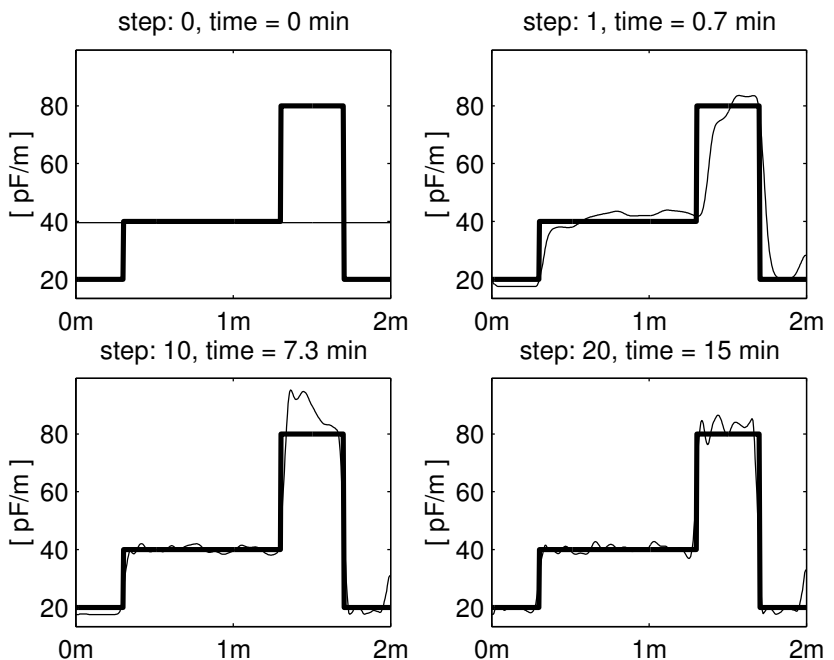

Fig. 8. Capacitance profiles $C(x)$ during the reconstruction from left-sided reflection data (thin line) for lossless material $(G(x) \equiv 0 \mathrm{mS} / \mathrm{m})$ compared to the true profile (bold line).
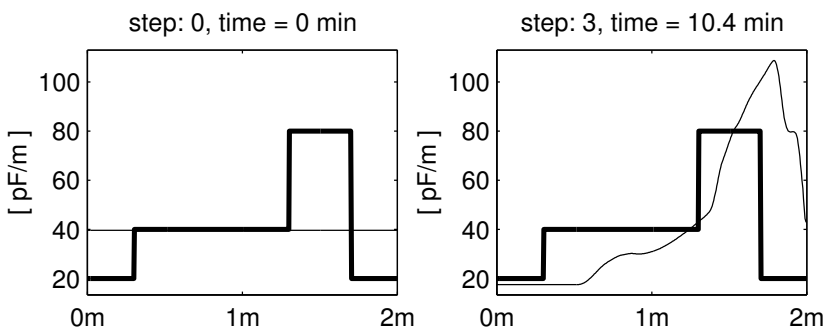

Fig. 9. Capacitance profiles $C(x)$ during the reconstruction from left-sided reflection data (thin line) for lossy material (wrong assumption of $G(x) \equiv 0 \mathrm{mS} / \mathrm{m}$ during reconstruction) compared to the true profile (bold line).

sides of the cable sensor are used. The minimization of Eq. (23) requires the determination of the solution of two independent IBVP. In addition to this duplication of the calculation effort the conjugate gradient method is more complex. To find the optimal step sizes for each search direction a twodimensional search has to be treated. This causes a much larger calculation time for every iteration step. To keep the total calculation time acceptable, the terminating condition to exit the conjugate gradient method has to be less strict.

Figure 11 shows the result of the optimization during several iteration steps. The algorithm terminates after 8 iteration steps although the approximation to the true profile is not as good as for the single-sided reflection data. But in addition to the capacitance profile the algorithm leads to a conductivity profile (see Fig. 12).

This conductivity profile represents the total conductance of the composite of insulation and surrounding soil. A transformation from $G(x)$ to the soil conductivity $\sigma(x)$ is not required because it gives no further information to the water content. But further investigations may close this gap.
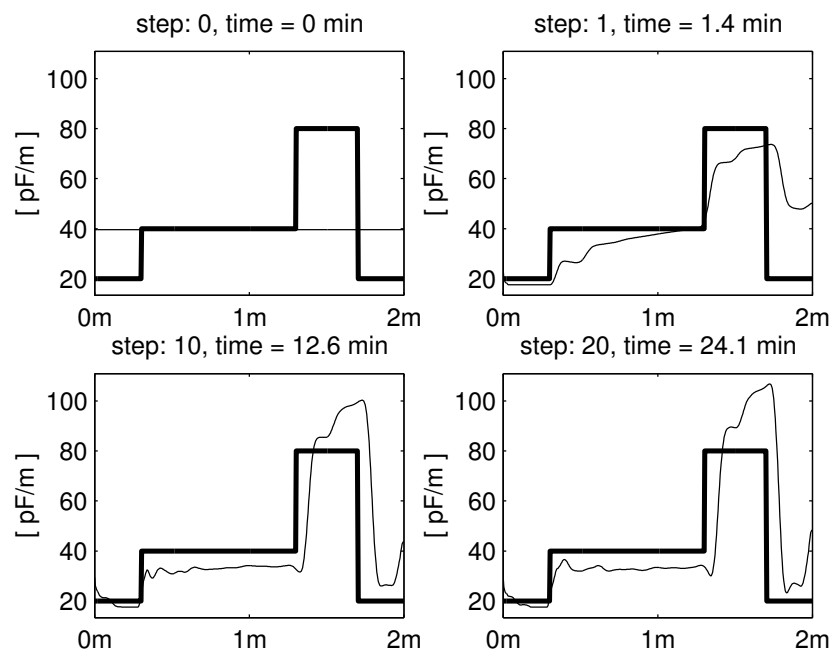

Fig. 10. Capacitance profiles $C(x)$ during the reconstruction from left-sided reflection data (thin line) for lossy material (assumption of constant $G(x) \equiv 4 \mathrm{mS} / \mathrm{m}$ during reconstruction) compared to the true profile (bold line).
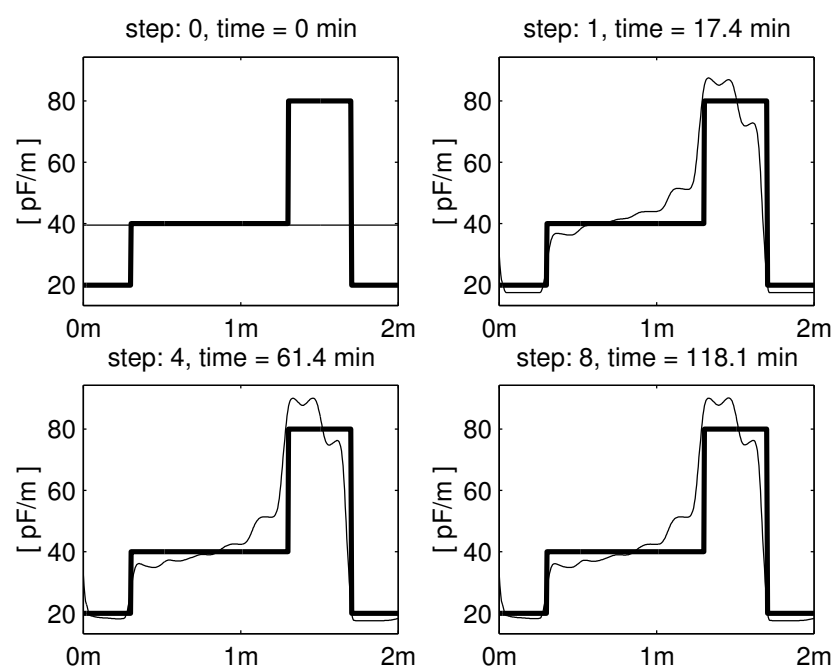

Fig. 11. Capacitance profiles $C(x)$ during the reconstruction from double-sided reflection data (thin line) for lossy material compared to the true profile (bold line).

Finally the iteration speed and the total calculation time of the presented numerical examples were investigated. The results are presented in Fig. 13.

One can see that the one-parameter reconstruction for lossless material leads to a very good approximation of the given profile and that a quasi-steady state is reached after 27 iterations. But the conductivity profile is exactly known in advance. In the case of lossy material the best approximation to the true profile is given by the double-sided reconstruction. The value of the cost function is half as much than for the single-sided reconstruction with constant conductivity (keep 

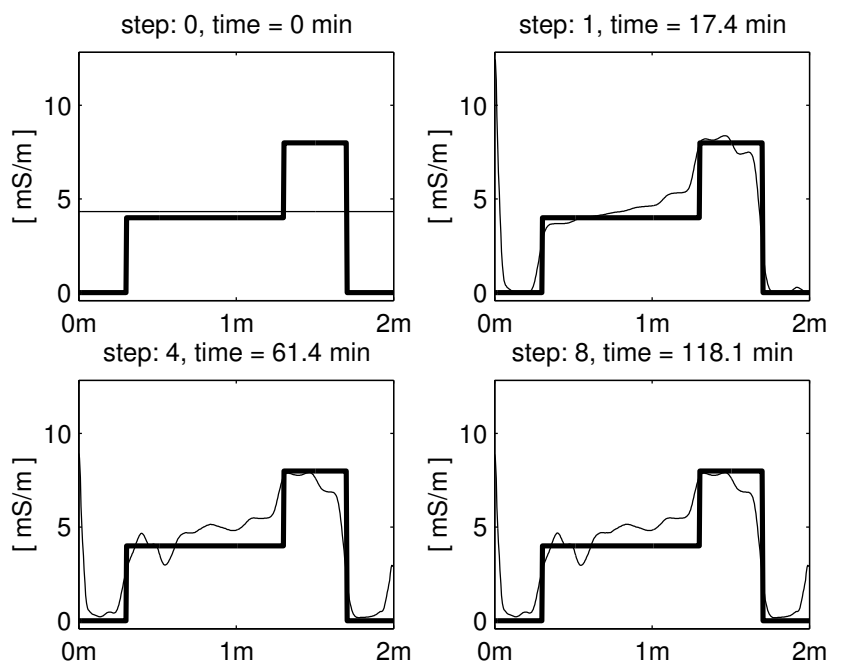

Fig. 12. Conductance profiles $G(x)$ during the reconstruction from double-sided reflection data (thin line) for lossy material compared to the true profile (bold line).

in mind the logarithmic scale of the y-axis in the upper diagram of Fig. 13).

The calculation effort stays constant in every iteration step and is nearly equal for the compared one-parameter reconstructions. It increases rapidly when using the two-parameter reconstruction due to the two-dimensional step size optimization. But this calculation time is almost fast enough for many applications and surely contributes to the further spreading of this method.

The software for this reconstruction algorithm has been developed using MATLAB ${ }^{\circledR}$. It has been employed to many applications of the SMG for different transmission lines between 0.3 and $20 \mathrm{~m}$ in length. Progress is intended to continue and the relevant software can be available shortly.

\section{Conclusions}

A fast inversion technique is presented that derives capacitance profiles in high spatial resolution from single TDR reflection measurements. The algorithm is based on an optimization approach to minimize the difference between the measurement and simulated TDR reflection data depending on a given parameter distribution. The optimization is done with conjugate gradients due to the fact that the gradient can be calculated explicitly. This gradient can be determined very fast by solving only two initial-boundary-valueproblems instead of several hundred when using standard Hessian matrix inversion techniques. The algorithm iterates very fast and leads to reliable soil moisture profiles which can be derived from the capacitance profiles by standard transformations.
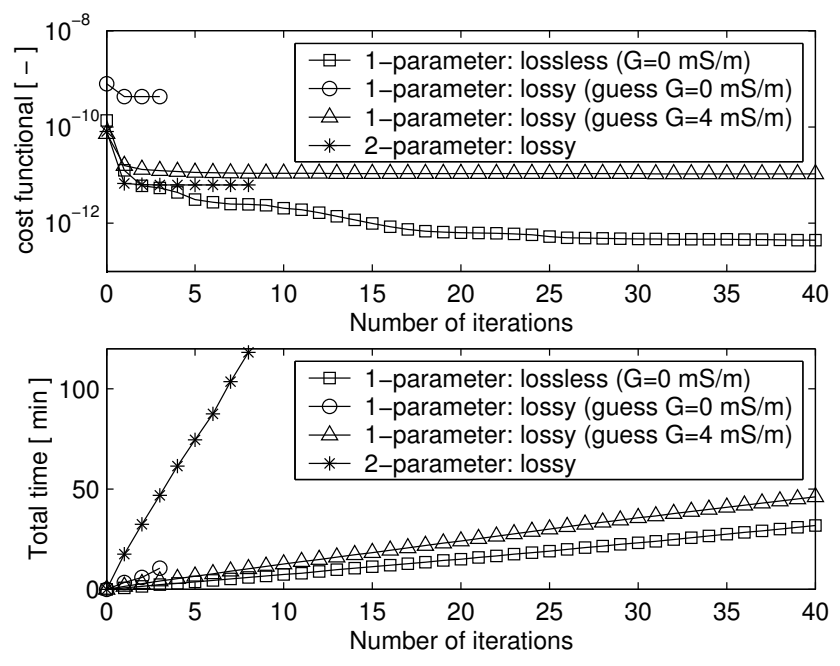

Fig. 13. Results of the cost function (above) and the total calculation time (below) after each iteration step of the cg-method.

The results of this study of this new inversion technique for time domain reflectometry data show that single-sided reflection data are capable for the reconstruction of the soil moisture profile for lossless (or low-loss) soils. The full information content of one single travel-time roundtrip can be used to determine the capacitance profile $C(x)$ and the associated volumetric water content on a standard PC in reasonable time. In the case of lossy soils more information is required. The knowledge of the conductance profile $G(x)$ or an experimental determined relationship between capacitance and conductance for the used sensor and soil can improve the determination of moisture profiles using only one single measurement. If none of this knowledge is available one more independent reflection measurement is required.

The presented inversion technique is also suitable for the simultaneous reconstruction of capacitance and conductance profile using double-sided reflection data. The resulting profiles are more reliable than single-sided reconstructions with standard assumptions to the conductivity (e.g. constant conductivity distributions). The simultaneous reconstruction of $C(x)$ and $G(x)$ and the associated volumetric water content can also be done within a reasonable time.

Edited by: N. Romano

\section{References}

Becker, R.: Spatial time domain reflectometry for monitoring transient soil moisture profiles, Ph.D. thesis, Institut für Wasser und Gewässerentwicklung - Bereich Wasserwirtschaft und Kulturtechnik, Mitteilungen Heft 228, Universität Karlsruhe, 2004.

Becker, R. and Hübner, C.: Messgeräteentwicklungen der Soil Moisture Group, in Kolloquium mit Workshop "Innovative 
Feuchtemessung in Forschung und Praxis”, Karlsruhe, Germany, 3-4 July 2003.

Becker, R. and Schlaeger, S.: Spatial time domain reflectometry with rod probes, Proceedings of the 6th Conference on "Electromagnetic Wave Interaction with Water and Moist Substances", ISEMA 2005, Weimar, Germany, 20 May-1 June 2005.

Becker, R., Bieberstein, A., Hübner, C., Nüesch, R., Schädel, W., Scheuermann, A., Schlaeger, S., and Schuhmann, R.: Nondestructive in situ and online measurements of soilphysical parameters, in Soil and Rock America 2003, 12th Panamerican Conference on Soil Mechanics and Geotechnical Engineering, 39th US Rock Mechanics Symposium, Cambridge, 22-26 June, 2003.

Becker, R., Brandelik, A., Hübner, C., Schädel, W., Scheuermann, A., and Schlaeger, S.: Soil and snow moisture measurement system with subsurface transmission lines for remote sensing and environmental applications - Results of the Soil Moisture Group of the University of Karlsruhe, in Proceedings of the Open Symposium on Propagation and Remote Sensing, URSI CommissionF, Garmisch-Partenkirchen, Germany, 12-14 February 2002.

Birchak, J. R., Gardner, C. G., Hipp, M. E., and Victor, J. M.: High dielectric constant microwave probes for sensing soil moisture, Proceedings of the IEEE, 62, 93-98, 1974.

Brandelik, A., Huebner, C., and Schuhmann, R.: Moisture sensor for large area layers (German patent no. 4432687, European patent no. 0804724, US patent no. 5942904), 16 June 1998.

Box, M. J.: A new method of constraint optimization and a comparison with other methods, The Computer Journal, 8, 43-52, 1965.

Chambarel, A., Ferry, E., Chanzy, A., Laurent, J.-P., Todoroff, P., and Ferrari: P.: TDR signal modeling using the electric line approach: model validation and signal inversion to retrieve soil moisture profile, TDR 2001, Evanston, Illinois, 5-7 September 2001.

Dasberg, S. and Dalton, F. N.: Time domain reflectometry field measurements of soil water content and electrical conductivity, Soil Sci. Soc. Am. J., 49, 293-297, 1985.

Engquist, B. and Majda, A.: Absorbing boundary conditions for the numerical simulation of waves, Mathematics of Computation, 31, 629-651, 1997.

Feng, W., Lin, C. P., Deschamps, R. J., and Drnevic, V. P.: Theoretical model of a multisection time domain reflectometry measurement system, Water Resources Research, 35, 8, 2321-2331, 1999.

Fletcher, R. and Reeves, C. M.: Function minimization by conjugate gradients, The Computer Journal, 7, 149-154, 1964.

Hakansson, G: Reconstruction of soil moisture profile using timedomain reflectometer measurements, Master thesis, Royal Institute of Technology, Department of Electromagnetic Theory, Stockholm, 1997.

He, S., Kabanikhin, S. I., Romanov, V. G., and Ström, S.: Analysis of the Green's function approach to one-dimensional inverse problems, Part I: One parameter reconstruction, J. Math. Phys., 34, 5724-5746, 1993.

He, S., Romanov, V. G., and Ström, S.: Analysis of the Green's function approach to one-dimensional inverse problems, Part II: Simultaneous reconstruction of two parameters, J. Math. Phys., 35, 2315-2335, 1994.

Heimovaara, T. J. and Bouten, W.: A computer-controlled 36channel time domain reflectometry system for monitoring soil water content, Water Resources Research, 26, 2311-2316, 1990.

Heimovaara, T. J., Huismann, J. A., Vrugt, J. A., and Bouten, W. Obtaining the spatial distribution of water content along a TDR probe using the SCEM-UA Bayesian inverse modelling scheme, Vadose Zone Journal, 3, 1128-1145, 2004.

Hook, W. R., Livingston, N. J., Sun, Z. J., and Hook, P. B.: Remote diode shorting improves measurement of soil water by time domain reflectometry, Soil Sci. Soc. Am. J., 56, 1384-1391, 1992.

Hübner, C.: Entwicklung hochfrequenter Messverfahren zur Boden- und Schneefeuchtebestimmung, Ph.D. thesis, 199 pp., Forschungszentrum Karlsruhe, Institute for Meteorology and Climate Research, Scientific Report FZKA 6329, Karlsruhe, 1999.

Hübner, C., Schlaeger, S., Becker, R., Scheuermann, A., Brandelik, A., Schädel, W., and Schuhmann, R.: Advanced measurement methods in time domain reflectometry for soil moisture determination, in: Electromagnetic Aquametry, edited by: Kupfer, K., Springer, Berlin, Heidelberg, New York, 2005.

Lundstedt, J. and He, S.: A time-domain optimization technique for the simultaneous reconstruction of the characteristic impedance, resistance and conductance of a transmission line, J. Electromagn. Waves and Appl., 10, 581-602, 1996.

Nelder, J. A. and Mead, R.: A simplex method for function minimization, The Computer Journal, 7, 308-313, 1965.

Norgren, M. and He, S.: An Optimization approach for the frequency-domain inverse problem for a nonuniform LCRG transmission line, IEEE Transaction on Microwave Theory and Technique, 44, 1503-1507, 1996.

Oswald, B.: Full wave solution of inverse electromagnetic problems, Ph.D. thesis Swiss Federal Institute of Technology, Zürich, 2000.

Pereira, D. S.: Développement d'une nouvelle méthode de détermination des profils de teneur en eau dans les sols par inversion d'un signal TDR, Ph.D. thesis, Lab. D'Etude des Transf. En Hydrol. Et Environ., (LTHE), Univ. Joseph Fourier-Grenoble I, Grenoble, France, 1997.

Polak, E. and Ribière, G.: Note sur la convergence de methodes de directions conjugees, Revue Française d'Informatique et de Recherche Operationelle, Serie Rouge, 3, 35-43, 1969.

Robinson, D. A., Jones, S. B., Wraith, J. M., Or, D., and Friedman, S. P.: A review of advances in dielectric and electrical conductivity measurement in soils using time domain reflectometry, Vadose Zone Journal, 2, 444-475, 2003.

Scheuermann, A., Schlaeger, S., Hübner, C., Brandelik, A., and Brauns, J.: Monitoring of the spatial soil water distribution on a full-scale dike model, in Proceedings of the Fourth International Conference on Electromagnetic Wave Interaction with Water and Moist Substances, Weimar, MFPA, 343-350, 2001.

Schlaeger, S.: Inversion von TDR-Messungen zur Rekonstruktion räumlich verteilter bodenphysikalischer Parameter, Ph.D. thesis, Veröffentlichungen des Institutes für Bodenmechanik und Felsmechanik der Universität Fridericiana in Karlsruhe, vol. 156, 189 pp., Karlsruhe, Germany, 2002.

Stacheder, M., Hübner, C., Schlaeger, S., and Brandelik, A,: Combined TDR and low frequency permittivity measurements for continuous snow wetness and snow density determination, in: Electromagnetic Aquametry, edited by: Kupfer, K., Springer, Berlin, Heidelberg, New York, 2005. 
Todoroff, P., Lorion, R., and Lan Sun Luk, J. D.: L'utilisation des algorithmes génétiques pour l'identification de profil hydriques de sol a partir de courbes réflectrométriques, C.R. Acad. Sci. Ser. IIa, Sci. Terre Planetes, 327, 607-610, 1998.

Todoroff, P. and Lan Sun Luk, J. D.: Calculation of in situ soil water content profiles from TDR signal traces, Measurement Science and Technology, 12, 27-36, 2001.

Topp, G. C. and Davis, J. L.: Measurement of soil water content using time-domain reflectometry (TDR): A field evaluation, Soil Sci. Soc. Am. J., 49, 19-24, 1985.

Topp, G. C., Davis, J. L., and Annan, A. P.: Electromagnetic determination of soil water content. Measurements in coaxial transmission lines, Water Resources Research, 16, 574-582, 1980.
Topp, G. C., Davis, J. L., and Annan, A. P.: Electromagnetic determination of soil water content using TDR: I Applications to wetting fronts and steep gradients, Soil Sci. Soc. Am. J., 46, 672678, 1982a.

Topp, G. C., Davis, J. L., and Annan, A. P.: Electromagnetic determination of soil water content using TDR: II Evaluation of installation and configuration of parallel transmission lines, Soil Sci. Soc. Am. J., 46, 678-684, 1982b.

Wraith, J. M., Robinson, D. A., Jones, S. B., and Long, D. S.: Spatially characterizing apparent electrical conductivity and water content of surface soils with time domain reflectometry, Computers and Electronics in Agriculture, 46, 239-261, 2005. 